(2) Open Access Full Text Article

\title{
Intestinal metastasis from breast invasive ductal carcinoma after a long latency: case report and literature review
}

This article was published in the following Dove Press journal: OncoTargets and Therapy

\author{
Min Liu' \\ Lei Zhang ${ }^{2}$ \\ Liang Guo ${ }^{3}$ \\ Jincai $L^{\prime}$ \\ Weiyan Shi' \\ Bailong Liu' \\ 'Department of Radiation Oncology, \\ The First Hospital, Jilin University, \\ Changchun I3002I, China; \\ ${ }^{2}$ Department of Radiology, The First \\ Hospital, Jilin University, Changchun \\ I3002 I, China; ${ }^{3}$ Department of \\ Pathology, The First Hospital, Jilin \\ University, Changchun I3002I, China
}

Correspondence: Bailong Liu Department of Radiation Oncology, The First Hospital, Jilin University, 7I Xinmin Street, Changchun I3002I, China

Tel +86 I58 04302752

Email liubl@jlu.edu.cn

\begin{abstract}
Intestinal metastasis from breast cancer (BC) is rarely encountered in clinical practice. Nonspecific symptoms and long latency result in misdiagnosis as a primary intestinal tumor. Therefore, increased awareness of bowel metastasis secondary to BC and a thorough understanding of the clinical and molecular features, and intervention of bowel metastasis are fundamental to avoid the delay of correct diagnosis and management. Herein, we documented a BC patient who experienced progressive bellyache and vomiting 16 years after simplified radical mastectomy. Abdominal CT scan revealed localized thickening of the small intestine wall and lumen narrowing, initially diagnosed as a primary intestinal tumor. The subsequent operation resolved the intestinal obstruction and confirmed the diagnosis of intestinal involvement of BC. Radical local treatment followed by systemic intervention contributed to a better outcome. Our case indicates that intestinal metastasis should be included in the diagnostic checklist in patients presented with any intestinal symptom even with a remote history of BC. Our case is of great value in its rarity and calls for the awareness of clinicians for this special entity to guarantee the accurate and prompt diagnosis and treatment, and optimize the patient's prognosis.
\end{abstract}

Keywords: intestinal metastasis, breast cancer, surgery, combined treatment

\section{Introduction}

Breast cancer $(\mathrm{BC})$, the most common cancer in females globally, threats to women's health seriously as the leading cause of mortality. Since 1991, with the improvement of early detection and treatment strategies, the mortality of $\mathrm{BC}$ has been declining. ${ }^{1-3}$ With longer survival, more patients experience recurrence and metastasis. Common metastatic sites of BC include lung, liver, skeleton, and brain. Invasive lobular carcinoma (ILC) of breast shows a predilection for uncommon metastatic sites such as gastrointestinal (GI) tract, peritoneum and uterus, ovary, and skin. ${ }^{4}$ The differentiation of bowel metastasis from $\mathrm{BC}$ and primary intestinal tumor is difficult since there are vague, diverse symptoms and long disease-free duration of $\mathrm{BC}$. We presented a bowel involvement in a female diagnosed with invasive ductal BC 16 years ago. This rare case report and related literature review help us to extend our understanding of this special condition and guarantee such patients prompt, correct treatment and a better outcome.

\section{Case report}

A 56-year-old Chinese female was admitted to a tertiary hospital in February 2000 for painless mass in left breast. Left simplified radical mastectomy was performed 
and the postoperative pathology demonstrated invasive ductal breast carcinoma with immunohistochemical results of $\mathrm{ER}(+)$ and $\mathrm{PR}(+)$ and left axillary lymph node involvement (4/6). Then, she underwent six cycles of adjuvant chemotherapy with Cyclophosphamide, 5-fluorouracil, Epirubicin, and local radiotherapy. Tamoxifen was taken from December 2000 to December 2004. In June 2010, she complained of left frontal mass. The subsequent surgery manifested metastatic adenocarcinoma of left frontal bone with immunohistochemical results of ER(2+), PR(3+), and HER-2(-) identical to the breast origin. Letrozole was taken for the following 6 years.

In May 2016, the patient was referred to our hospital for 4-month reduced exhaust defecation and 2-month progressive abdominal pain with nausea and vomiting. On physical examination, the abdomen was distended with mild tenderness and no rebound pain. Bowel sounds were hyperactive with about 10/minute and over-water sound could be heard. The subsequent abdominal CT scan with contrast revealed part of the small intestine thickened with narrowing lumen and marked enhancement after contrast. The intestinal loops above the lesion dilated with effusion. Exploratory laparotomy was performed. During the operation, a circumferential tumor was found in the small intestine that involved the ileocecus with passive expansion of proximal intestine. Radical excision was carried out. The tumor was $2.5 \times 2 \times 1.5 \mathrm{~cm}$ and infiltrated the whole layers of the small intestine and appendiceal serosal layer (Figure 1). Two of 10 periintestinal lymph nodes were metastatic. All the margins were tumor-free. Immunohistochemically, the neoplastic cells were positive to estrogen receptor (ER), gross cystic disease fluid protein 15 (GCDFP-15), CA153, and cytokeratin (CK)7 while negative to CK20, Villin, and progesterone receptor (PR) which supported the bowel metastasis of $\mathrm{BC}$ (Figure 2A-G). Ki-67 expressed in the nuclei of approximately $10 \%$ of tumor cells (Figure $2 \mathrm{H}$ ). Reexamination of head MRI revealed multiple bone destruction in left frontal bone, temporal bone, and lateral orbital wall (Figure 3A and B). The patient recovered uneventfully and then she was enrolled in the clinical trial of Cedaramine CDM301 combined with Exemestane for hormone receptor (HR) positive BC. In January 2018, the skull involvement progressed (Figure $3 \mathrm{C}$ and $\mathrm{D}$ ) and she underwent local radiotherapy of $56 \mathrm{~Gy} / 28 \mathrm{f}$ with a stable disease response. Systemic screening showed no signs of metastasis except skull lesions. The endocrine therapy was switched to fulvestrant. Now, the patient is still alive with isolated skull metastasis.
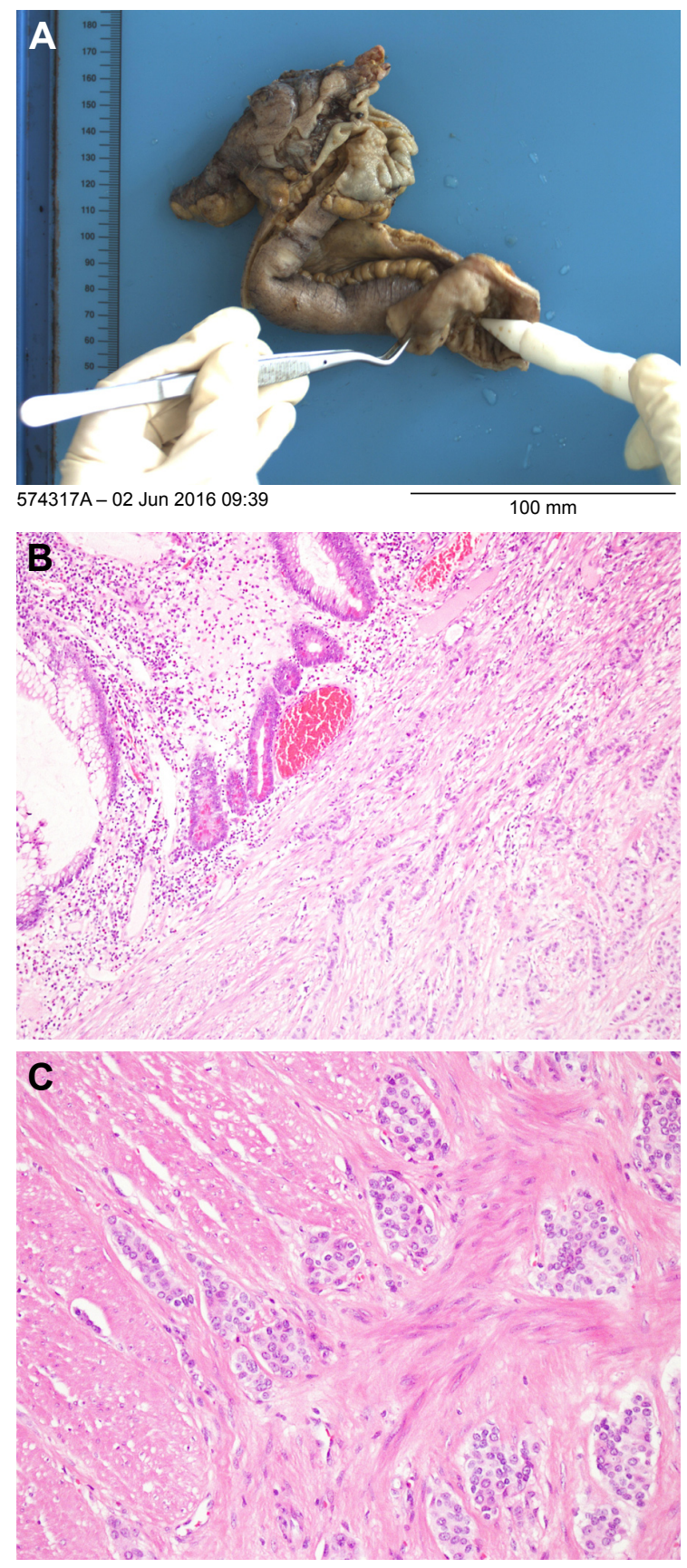

Figure I Pathological results of the resected bowel deposit.

Notes: (A) The postoperative gross specimen showed a circumferential lesion located in the small intestine measuring about $2.5 \times 2 \times 1.5 \mathrm{~cm}$ and causing the proximal bowel dilation. The cut surface of the tumor is grayish white, solid, and hard. (B) Hematoxylin-eosin stained section revealed submucous invasion of cancer cells (original magnification: 100x). (C) Hematoxylin-eosin stained section documented neoplastic cells infiltrated muscular layer exhibiting nest-like pattern (original magnification: 200x).

The study was approved by the Ethics Committee of the First Hospital of Jilin University, and written informed consent was obtained from the patient for publication of this case report and accompanying images. A copy of the written 

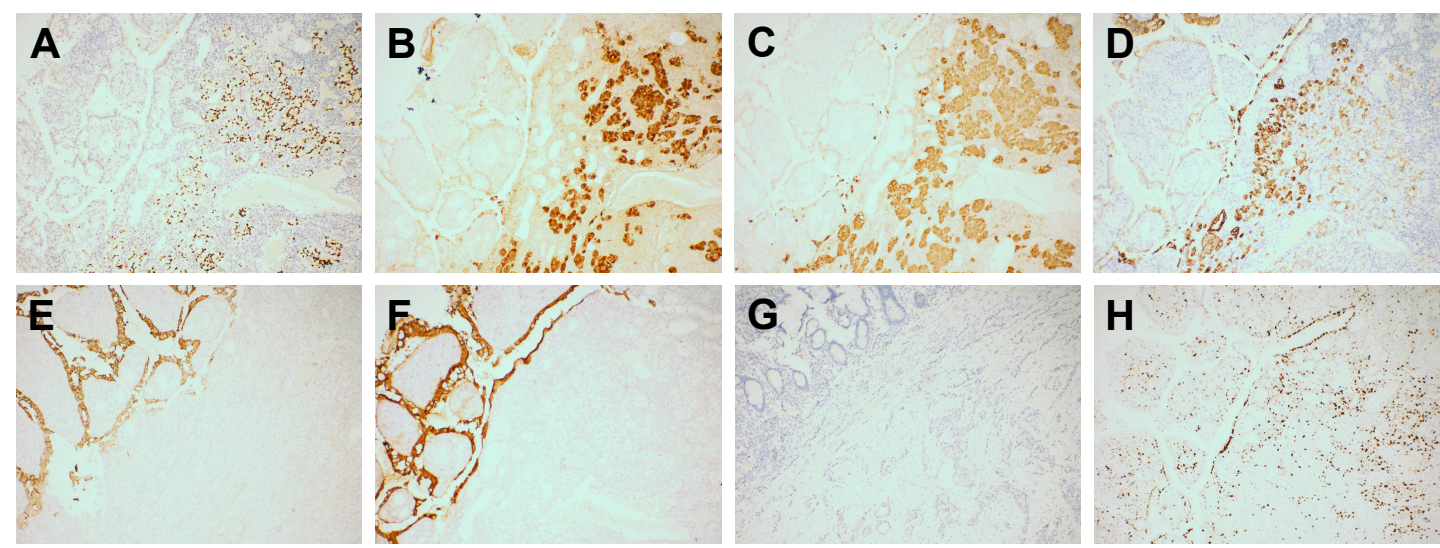

Figure $2 \mathrm{IHC}$ results suggestive of bowel metastasis of $\mathrm{BC}$ origin (upper left part: normal intestinal mucosa; lower right part: tumor infiltrating part.)

Notes: (A-C) ER, GCDFP-15, and CA153 were intensively positive for tumor cells (original magnification: 100x). (D) CK7 was partly positive for tumor cells (original magnification: 100x) while (E-G) CK20, Villin, and PR were negative for neoplastic cells (original magnification: 100x). (H) Ki-67 expressed in the nuclei of $\sim 10 \%$ of tumor cells (original magnification: 100x)

Abbreviations: BC, breast cancer; ER, estrogen receptor; IHC, immunohistochemistry; PR, progesterone receptor.
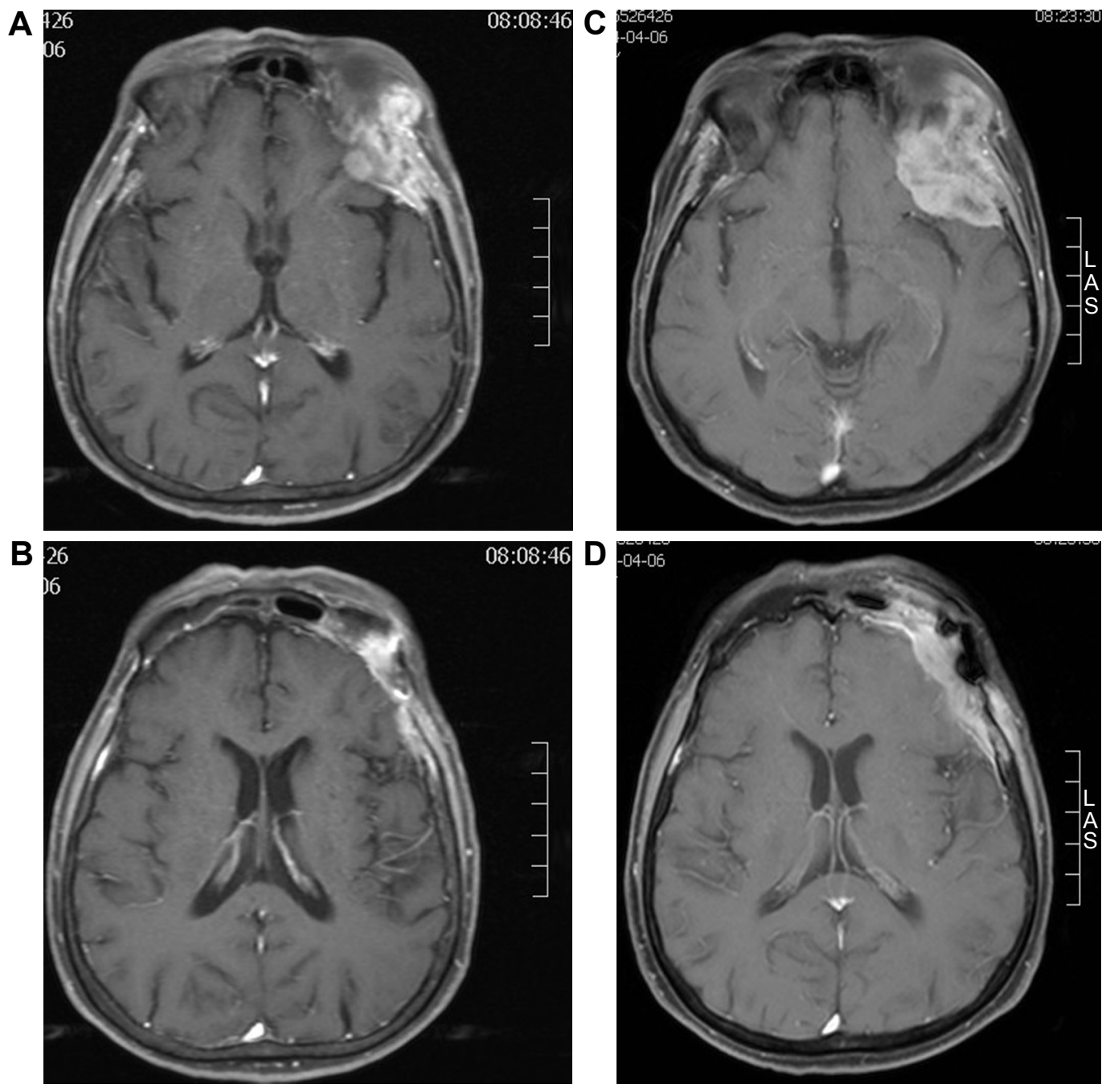

Figure 3 Selective cranial MRI scan images of skull involvement.

Notes: (A, B) Head MRI of July 2016 revealed multiple bone destruction in left frontal bone, temporal bone, and lateral orbital wall. (C, D) In January 20I8, the skull metastasis progressed with obvious localized soft tissue lesions. 
consent is available for review with the Editor-in-Chief of this journal.

\section{Discussion}

The alimentary tract metastasis from BC is relatively infrequent. The most commonly involved segments are colon and rectum, followed by stomach, then small intestine..$^{5}$ ILC, constituting only $12 \%$ of primary $\mathrm{BC}$, contributed to $64 \%$ GI metastases in the report by McLemore et al. ${ }^{5}$ Less are those originating from invasive ductal carcinoma (IDC) of breast as our case did. The concrete mechanisms for different spread patterns between ILC and IDC are still unclear. ILC usually lacks expression of E-cadherin, a key molecule in cell-to-cell adhesion, which might give rise to this GI predilection partly. ${ }^{4,6}$

The early diagnosis of bowel metastasis from BC is quite challenging for several reasons. Patients complain of bellyache, change in bowel habits, nausea, vomiting, unintentional weight loss and fatigue, common in both primary and secondary intestinal tumors. In BC individuals, GI metastasis can occur after a long-term latency up to 30 years. ${ }^{7}$ However, it can also be the first sign of BC. ${ }^{8-11}$ Sometimes, acute abdominal syndromes such as bowel obstruction due to metastasis lead to the diagnosis of primary BC. ${ }^{10,12-15}$ The long disease-free interval, like our patient, of 16 years or unknown history of $\mathrm{BC}$ poses great difficulty to the established diagnosis of bowel metastasis. López Deogracias et al documented an ILC patient who developed rectal metastasis causing urethral dilation after a 15 -year latency. ${ }^{16}$ Intestinal metastasis can originate from male BC. Ferrari et al described an extremely rare case of duodenal metastasis from IDC of breast in an elderly male 40 months after breast surgery. ${ }^{17}$ Koleilat et al documented a male ductal BC developed colon metastasis causing mechanical bowel obstruction after a duration of more than 10 years. ${ }^{18}$ All these caution us that a detailed medical history inquiry is crucial for any patient referred for digestive complaints especially for those with $\mathrm{BC}$ history, and intestinal metastasis from $\mathrm{BC}$ should be included in the diagnostic checklist.

For early stage of metastasis, endoscopy generally reveals intact and normal mucosa since metastasis often involved the submucosal layer. Therefore, superficial biopsy is usually inconclusive. In the case of metastatic $\mathrm{BC}$ simulating colon linitis plastica reported by Okido et al, the biopsy showed necrotic tissue and failed to find carcinoma. The postoperative histological findings confirmed the colon metastasis from breast ILC. ${ }^{19}$ Carcoforo et al documented a 73 -year-old women referred with abdominal pain, nausea, and emesis without oncologic history. Colonoscopy demonstrated a stricture $15 \mathrm{~cm}$ above the anal verge. Multiple biopsies were nondiagnostic while exploratory laparotomy with biopsy contributed to the diagnosis of late ILC intestinal metastases. Subsequently, the primary ILC of left breast was unveiled. ${ }^{13}$ Deep or bite biopsy and surgery are helpful for the prompt and correct diagnosis of bowel metastasis. In the late setting, the metastatic lesion can invade the entire bowel layers which manifest as ulcer, bleeding by endoscopy, mimicking the intestinal malignant primacy. The intestinal foci can be single or multiple. Sironi et al described a case in which three metastatic foci were located in right, transverse, and left colon from an asymptomatic BC. ${ }^{20}$

Radiologically, stricture or linitis plastica is a typical manifestation by barium examination. As the disease progresses, the involved bowel wall becomes diffuse thickening and rigid, causing stenosis and obstruction. ${ }^{19,21,22}$ Lack of dysplasia in adjacent bowel epithelium is a clue for intestinal metastasis. ${ }^{23}$ The direct pathologic comparison of the resected or biopsy bowel specimen with the primary BC specimen will be helpful for the accurate diagnosis. ${ }^{19}$

Immunohistochemical staining is critical in the correct diagnosis. GCDFP-15, ER, and/or PR are strongly immunoreactive in metastatic BC. CK7 expression and CK20 negativity are common in IDC of breast $\mathrm{t}^{19}$ as presented in our case. Villin is a normal intestinal epithelial cell labeling. Villin negativity is suggestive of bowel metastasis of other origin.

GI metastasis is generally a sign of disseminated disease. ${ }^{23}$ Systematic screening is necessary to identify other metastatic deposits. For isolated GI involvement, radical resection followed by medical intervention might contribute to a better prognosis. Our patient developed oligometastasis in skull and bowel, both taking local radical management and updated systematic treatment. The first surgery of skull bone metastasis resulted in a 6-year disease-free survival (DFS). The second excision of bowel metastasis led to a 2-year local relapse-free survival. Therefore, for individuals of limited metastases and good performance status, aggressive local modalities such as surgery and/or radiotherapy combined with chemotherapy and/or endocrine strategies are still promising for long-term survival. In the case described by Rajan et al, a 60-year-old female diagnosed as having IDC 9 years ago experienced rectal metastasis without signs of other organ dissemination. A short course of preoperative radiotherapy followed by surgery contributed to a 4-year DFS. ${ }^{24}$ Similarly, in an ILC patient who experienced colon metastasis of hepatic flexure after a 12-year latency, right hemicolectomy combined with treatment including vinorelbine and letrozole attained a 20-month overall survival 
with no evidence of relapse. ${ }^{25}$ Calò et al presented a solitary jejunal metastasis from an undiagnosed ILC case. Surgeries to both the breast primary and jejunal metastasis followed by aromatase inhibitor gained a 29-month PFS. ${ }^{12}$ HR expression has a positive effect on survival. ${ }^{15}$ Multiple endocrine drugs and antiresistant drugs such as CDK4/6 and m-TOR inhibitors are good options. Nevertheless, surgery should be performed in case of perforation, bleeding, or intestinal obstruction to avoid severe complications and improve the quality of life.

\section{Conclusion}

Intestinal metastasis should be considered when dealing with patients of any intestinal symptom especially for those with a BC history. Work-up including systemic screening, deep biopsy or resection, immunohistochemical staining, and a thorough pathological comparison of the bowel and primary $\mathrm{BC}$ specimen is fundamental for the correct diagnosis and staging. For isolated bowel metastasis, radical local treatment followed by systemic intervention might attain a better survival. The optimal strategy for this special entity needs to be clarified by large-scale clinical trials in the near future.

\section{Disclosure}

The authors report no conflicts of interest in this work.

\section{References}

1. Early Breast Cancer Trialists' Collaborative Group (EBCTCG). Effects of chemotherapy and hormonal therapy for early breast cancer on recurrence and 15-year survival: an overview of the randomised trials. Lancet. 2005;365(9472):1687-1717.

2. Siegel RL, Miller KD, Jemal A. Cancer statistics. CA Cancer J Clin. 2015; 2015(65):5-29.

3. Berry DA, Cronin KA, Plevritis SK, et al. Effect of screening and adjuvant therapy on mortality from breast cancer. $N$ Engl J Med. 2005; 353(17):1784-1792

4. Sastre-Garau X, Jouve M, Asselain B, et al. Infiltrating lobular carcinoma of the breast. Clinicopathologic analysis of 975 cases with reference to data on conservative therapy and metastatic patterns. Cancer. 1996; 77(1):113-120.

5. Mclemore EC, Pockaj BA, Reynolds C, et al. Breast cancer: presentation and intervention in women with gastrointestinal metastasis and carcinomatosis. Ann Surg Oncol. 2005;12(11):886-894.

6. Critchley AC, Harvey J, Carr M, Iwuchukwu O. Synchronous gastric and colonic metastases of invasive lobular breast carcinoma: case report and review of the literature. Ann R Coll Surg Engl. 2011;93(5):e49-e50.

OncoTargets and Therapy

\section{Publish your work in this journal}

OncoTargets and Therapy is an international, peer-reviewed, open access journal focusing on the pathological basis of all cancers, potential targets for therapy and treatment protocols employed to improve the management of cancer patients. The journal also focuses on the impact of management programs and new therapeutic agents and protocols on
7. Schwarz RE, Klimstra DS, Turnbull AD. Metastatic breast cancer masquerading as gastrointestinal primary. Am J Gastroenterol. 1998; 93(1):111-114.

8. Arrangoiz R, Papavasiliou P, Dushkin H, Farma JM. Case report and literature review: metastatic lobular carcinoma of the breast an unusual presentation. Int J Surg Case Rep. 2011;2(8):301-305.

9. Mouawad NJ, Cleary RK. Small bowel obstruction as the primary presentation of undiagnosed metastatic lobular breast carcinoma. Breast Dis. 2011;33(1):35-40.

10. Sato T, Muto I, Hasegawa M, et al. Breast signet-ring cell lobular carcinoma presenting with duodenal obstruction and acute pancreatitis. Asian J Surg. 2007;30(3):220-223.

11. Tsujimura K, Teruya T, Kiyuna M, et al. Colonic metastasis from breast carcinoma: a case report. World J Surg Oncol. 2017;15(1):124.

12. Calò PG, Fanni D, Ionta MT, Medas F, Faa G, Atzori F. Jejunal obstruction caused by metastasis from an undiagnosed breast cancer: a case report. Tumori. 2012;98(3):89-91.

13. Carcoforo P, Raiji MT, Langan RC, et al. Infiltrating lobular carcinoma of the breast presenting as gastrointestinal obstruction: a mini review. $J$ Cancer. 2012;3:328-332.

14. Kandil E, King S, Alabbas H, Moroz K, Wright M. Image of the month. Small-bowel metastasis from infiltrating lobular breast cancer. Arch Surg. 2009;144(10):973-974.

15. Savanis G, Simatos G, Tzaida O, et al. Gastrointestinal tract metastasis as first presentation of breast cancer. $J$ BUON. 2006;11(1):79-81.

16. López Deogracias M, Flores Jaime L, Arias-Camisón I, et al. Rectal metastasis from lobular breast carcinoma 15 years after primary diagnosis. Clin Transl Oncol. 2010;12(2):150-153.

17. Ferrari AB, Pulcini G, Gheza F, et al. Duodenal metastasis from male breast cancer: a case report and review of the literature. J Med Case Rep. 2009;3:8331.

18. Koleilat I, Syal A, Hena M. Metastatic male ductal breast cancer mimicking obstructing primary colon cancer. Int J Biomed Sci. 2010; 6(1):66-70.

19. Okido M, Seo M, Hamada Y, et al. Metastatic breast carcinoma simulating linitis plastica of the colon: report of a case. Surg Today. 2011; 41(4):542-545.

20. Razzetta F, Tassara E, Saro F, Sironi M, D'Ambrosio G. Rare abdominal metastases from occult lobular breast cancer: report of two cases. Updates Surg. 2011;63(2):129-133.

21. Law WL, Chu KW. Scirrhous colonic metastasis from ductal carcinoma of the breast: report of a case. Dis Colon Rectum. 2003;46(10): 1424-1427.

22. Voravud N, El-Naggar AK, Balch CM, Theriault RL. Metastatic lobular breast carcinoma simulating primary colon cancer. Am J Clin Oncol. 1992;15(4):365-369.

23. Puglisi M, Varaldo E, Assalino M, Ansaldo G, Torre G, Borgonovo G. Anal metastasis from recurrent breast lobular carcinoma: a case report World J Gastroenterol. 2009;15(11):1388-1390.

24. Rajan S, Saeed M, Mestrah M. Ductal carcinoma of the breast metastasizing to the rectum. J Surg Case Rep. 2012;2012(5):12.

25. Signorelli C, Pomponi-Formiconi D, Nelli F, Pollera CF. Single colon metastasis from breast cancer: a clinical case report. Tumori. 2005; 91(5):424-427.

patient perspectives such as quality of life, adherence and satisfaction. The manuscript management system is completely online and includes a very quick and fair peer-review system, which is all easy to use. Visit http://www.dovepress.com/testimonials.php to read real quotes from published authors. 